\title{
Interaction of Staphylococcus aureus with osteoblasts (Review)
}

\author{
SIFENG SHI and XIANLONG ZHANG
}

\author{
Department of Orthopaedic Surgery, Shanghai Sixth People's Hospital, Jiao Tong University, Shanghai, P.R. China
}

Received August 29, 2011; Accepted November 22, 2011

DOI: $10.3892 /$ etm.2011.423

\begin{abstract}
Orthopedic infection is refractory to cure. Staphylococcus aureus (S. aureus) is the main causative pathogen responsible for orthopedic infection. $S$. aureus is capable of not only colonizing bone matrix, but also invading osteoblasts, which may play a significant role in the persistence and recurrence of osteomyelitis. Internalization requires the involvement of cytoskeletal elements, including actin microfilaments, microtubules and clathrin-coated pits. Microfilaments are most significant in the invasion process. $S$. aureus is capable of remaining alive in osteoblasts for a long period of time. Decreased sensitivity to antibiotics capable of penetrating host cells increases the difficulties of eradicating $S$. aureus. Osteoblasts, invaded by $S$. aureus, play a significant role in the initiation and maintenance of inflammatory immune responses. These osteoblasts recruit leukocytes and phagocytes to the site of inflammation via the expression of cytokines. Apoptosis is observed in osteoblasts invaded by $S$. aureus. Recruitment of osteoclasts and other immunocytes plays a crucial role in the resorption and destruction of bone.
\end{abstract}

\section{Contents}

1. Introduction

2. Invasion of osteoblasts by $S$. aureus

3. The fate and viability of $S$. aureus following invasion

4. The role of osteoblasts in the immune response

5. Apoptosis of osteoblasts following the internalization of S. aureus

6. Destruction of the bone due to inflammation and osteoclasts

7. Conclusion

\section{Introduction}

Orthopedic infection is a refractory disease that is associated with progression and recurrence (1). With increasing numbers

Correspondence to: $\operatorname{Dr}$ Xianlong Zhang, Department of Orthopedic Surgery, Shanghai Sixth People's Hospital, Jiao Tong University, 600 Yi Shan Road, 200233 Shanghai, P.R. China

E-mail: zhangxianlong20101@163.com

Key words: Staphylococcus aureus, osteoblast of foreign bodies implanted in orthopedic surgery, such as prosthetic joints, implant-related infection poses a threat to patients (2). Deep infection following arthroplasty is severe and thorough debridement and removal of the foreign body should be carried out (3).

Staphylococcus aureus (S. aureus) is the main causative pathogen responsible for orthopedic infection (4). S. aureus is capable of colonizing the matrix of the bone. This infection is difficult to eradicate since bacterial biofilm formation and bacteria embedded in biofilms become resistant to antibiotics, leading to the persistence of osteomyelitis (5). However, $S$. aureus was shown to invade osteoblasts, which may play a significant role in the persistence and recurrence of osteomyelitis (6-8).

The main function of osteoblasts is to synthesize bone matrix and regulate the activity of osteoclasts (9). However, the ability of $S$. aureus to be internalized by host cells and the expression of cytokines reveal the significant role of osteoblasts in the immune response.

\section{Invasion of osteoblasts by $S$. aureus}

Although not considered to be a typical intracellular pathogen, $S$. aureus was found to be capable of invading osteoblasts and other host cells in vitro $(2,4,10-15)$. Internalization of bacteria by osteoblasts also occurs in vivo (16). In one experiment, $S$. aureus were injected subcutaneously under the skin of the scalp and the allantoic sac of 17-day-old chick embryos. Following $48 \mathrm{~h}$, calvariae and tibiae were collected for transmission electron microscopy (TEM). S. aureus cells were found in approximately $14 \%$ of the calvarial osteoblasts following subcutaneous injection, and in $11 \%$ of calvarial and tibial osteoblasts following intra-allantoic injection (16). Internalization of $S$. aureus by osteoblasts have also been reported in a patient with recurrent, long-term osteomyelitis (17).

This phenomenon was considered to be another mechanism for the persistence and recurrence of chronic osteomyelitis. Bacteria and osteoblasts play a significant role in the invasion process. The attachment of $S$. aureus to osteoblasts is the first step of internalization and this attachment involves surface molecules of S. aureus. Fowler (18) proposed a model of attachment in which osteoblasts form a fibronectin bridge between surface-associated fibronectin-binding proteins of bacteria and host cell $\beta 1$ integrins; this bridge then leads to the invasion of S. aureus. Staphylococcus epidermidis (S. epidermidis) was also shown to be capable of invading bone cells. However, unlike $S$. aureus, S. epidermidis was unable to gain 
entrance into bone cells through a fibronectin bridge between the integrin and a bacterial adhesin (19).

Different $S$. aureus strains have variable capacity in internalizing bacteria. This capacity is found to be correlated with $\sigma$ B expressed by $S$. aureus (20). $\sigma \mathrm{B}$, the only $\sigma$ factor identified in $S$. aureus, is capable of prolonging the production of cell surface proteins, including fibronectin. $\sigma \mathrm{B}$ also plays a significant role in the regulation of virulence genes in $S$. aureus (21).

Internalization requires the involvement of cytoskeletal elements, including actin microfilaments, microtubules and clathrin-coated pits. Microfilaments are most significant in the invasion process (13). The process may be inhibited by monodansylcadaverine and cytochalasin D and, to some extent, by ouabain, monensin, colchicine and nocodazole (12). Notably, the invasion process does not require live bacteria (live and dead $S$. aureus are equally effective for invasion); however, live osteoblasts are required (22). The role of the osteoblast is positive in the process of invasion, and a 'zipper-type' mechanism has been proposed (23).

Calcium channels are also crucial in the invasion of osteoblasts, which are involved in the rearrangement of the cytoskeleton (24). The invasion of osteoblasts by $S$. aureus resulted in an increase in the phosphorylation of the extracellular signal-regulated protein kinases (ERK 1 and 2) (25). Activation of ERK 1 and 2 may result in the phosphorylation of numerous different substrates, including the transcription factors ATF-2, Elk-1 and c-Jun (26). ERK 1 and ERK 2 phosphorylation may also activate phospholipase A2, resulting in the production of leukotrienes (27). Leukotrienes may then open calcium channels on the host cell membrane (28).

\section{The fate and viability of $S$. aureus following invasion}

The fate of S. aureus following internalization by osteoblasts may correlate with the clinical manifestation of osteomyelitis. If $S$. aureus is capable of remaining alive in the intracellular environment, a thorough cure of osteomyelitis is difficult due to the difficulty of eradicating the bacteria in the osteoblast. Rifampin, chloramphenicol and clindamycin are the most active intracellular antibiotics. The majority of other antibiotics currently used are inactive intracellularly (e.g., lincomycin) or are incapable of penetrating cells (e.g., penicillins, cephalosporins and aminoglycosides) (29). Therefore, bacteria internalized by the osteoblast may be sequestered from the majority of antibiotics as well as the immune system.

Certain antibiotics are capable of eukaryotic cell penetration and have intracellular functions. However, similar to the bacteria embedded in the biofilms, the internalized bacteria may change their characteristics and decrease their sensitivity to antibiotics following the invasion of host cells and become situated in the intracellular environment. Investigators have used clindamycin and rifherampin to treat the $S$. aureus internalized in the osteoblast (30). The two antibiotics are capable of penetrating eukaryotic cells. Although the antibiotics may decrease or kill $S$. aureus internalized in the osteoblast at immediately following the invasion, the bacteria become less sensitive to the two antibiotics $12 \mathrm{~h}$ following the invasion (30). The observed structural changes were considered to be responsible for the change in sensitivity; however, metabolic change may also play a significant role. The change in sensitivity of $S$. aureus to antibiotics in the intracellular environment increases the difficulties of eradicating the pathogen.

Since $S$.aureus is capable of remaining alive in the osteoblast for a long period of time $(4,22)$, the bacteria released from the dead osteoblast may induce the recurrence of osteomyelitis (6). A previous study also showed the lack of decreased viability of S. aureus in invading a second osteoblast in vitro (31).

\section{The role of osteoblasts in the immune response}

As is commonly known, the primary roles of osteoblasts are to synthesize the components of the bone matrix and to regulate osteoclasts, which are bone resorption cells (32). However, there is increasing understanding of the function of osteoblasts in the initiation and maintenance of the inflammatory immune response. Osteocytes may recruit leukocytes and phagocytes to the site of inflammation via the expression of cytokines.

Cultured mouse and human osteoblasts infected with S. aureus were found to express high levels of interleukin (IL-6 and IL-12p75) (33). IL-12 is capable of stimulating T lymphocytes and natural killer (NK) cells to secrete significant amounts of interferon (IFN), activating macrophages and T lymphocytes to augment a Th1 response (33). Although IL-12 is known for its ability to protect against intracellular pathogens, it may contribute to the process of organ-specific autoimmune diseases (34). Monocyte chemoattractant protein-1 (MCP 1), produced by osteoblasts, also has the ability to recruit macrophages and certain $\mathrm{T}$ lymphocytes to areas of inflammation $(35,36)$. Osteoblasts may respond to bacterial infection by upregulating the expression of the chemokine CXCL10 (IP-10). IP-10 may then recruit $\mathrm{T}$ lymphocytes to the sites of bone infections (37). Dexamethasone, PGE(2) and T(h)2 cytokines are potential down-regulatory mediators of the chemokine (38).

Expression of NLRP3 in osteoblasts invaded by $S$. aureus was found in a study by McCall et al (39). The active NLRP3 inflammasome drives the innate immune response towards invading pathogens and cell damage, and regulates an adaptive immune response (40). The expression of NOD, a novel intracellular pattern recognition receptor, and Rip2 kinase, a critical downstream effector molecule for NOD signaling, was observed in osteoblasts invaded by $S$. aureus (41). NOD may regulate pro-inflammatory pathways in response to bacteria by inducing signalling pathways including nuclear factor $\kappa \mathrm{B}$ $(\mathrm{NF}-\kappa \mathrm{B})$ and mitogen-activated protein kinases (MAPKs) (42). However, certain authors believe it is the attachment, not invasion or secreted soluble factor(s) that activates $\mathrm{NF}-\kappa \mathrm{B}$ in human osteoblasts $(43,44)$.

\section{Apoptosis of osteoblasts following the internalization of S. aureus}

Osteoblasts invaded by few bacteria were found to be able to remain alive and differentiate into osteocytes (16). However, apoptosis or programmed cell death was found in osteoblasts invaded by $S$. aureus, but the process may also be induced by pathogens. Tucker et al (45) used light microscopy to examine morphological changes in the osteoblasts following the internalization of $S$. aureus. Cell rounding was observed, and dark centers, due to condensation of chromatin, were noted. Apoptotic nuclei were also present. 
Tumor necrosis factor-related apoptosis-inducing ligand (TRAIL) is crucial in the process of cell apoptosis. Alexander et al (8) examined the ability of $S$. aureus to induce the production of TRAIL by osteoblasts. Results demonstrated that $S$. aureus was capable of inducing TRAIL expression by osteoblasts. A dose-dependent response was observed $30 \mathrm{~min}$ following exposure to bacteria. Attachment of S. aureus to osteoblasts is necessary for optimal TRAIL induction (46). Messenger RNA (mRNA) molecules encoding TRAIL receptors were also expressed by osteoblasts. The expression of NLRP3 in osteoblasts invaded by $S$. aureus also provides a potential mechanism of apoptotic cell death of the host cells (39). Osteoblast apoptosis results in decreased matrix deposition and destruction of the bone (8).

\section{Destruction of the bone due to inflammation and osteoclasts}

Normal bone remodeling requires the coordinated regulation of the genesis and activity of osteoblast and osteoclast lineages (47). Therefore, apoptosis of osteoblasts is not the only cause of bone destruction. Recruitment of osteoclasts and other immunocytes play a significant role in the resorption and destruction of bone. In $S$. aureus infection, bone resorption is caused by proteins rather than lipoteichoic acid or muramyl dipeptide. The surface-assosiated protein fraction may stimulate fibroblasts or monocytes to release osteolytic cytokines and chemokines (48), including MCP-1, colony-stimulating factors (CSFs) and interleukins. Dexamethasone, $\mathrm{PGE}(2)$ and $\mathrm{T}(\mathrm{h}) 2$ cytokines have potential down-regulatory mediation of these chemokines (38).

Although MCP-1 is capable of recruiting macrophages and certain $\mathrm{T}$ lymphocytes to areas of inflammation $(35,36)$, $\mathrm{T}$ lymphocytes and macrophages may also be responsible for bone loss and contribute to the development of the progressive inflammatory damage (35). IL-6, produced by osteoblasts invaded by $S$. aureus, may directly or indirectly modulate the activity of osteoclasts, resulting in the induction of osteoclast differentiation or osteoclast-mediated bone demineralization (49). IL-6 expressed in the osteoblasts may be suppressed by certain agents including epigallocatechin gallate, a constituent of tea, which plays a role in the suppression of inflammation and decrease of bone resorption (50). IL-12 also contributes to the process of organ-specific autoimmune diseases (34). CSFs have a profound effect on osteoclastogenesis, high levels of granulocyte-macrophage-CSF (GM-CSF) and G-CSF secretion by osteoblasts challenged by $S$. aureus, and may induce osteoclastogenesis, resulting in bone resorption (51).

\section{Conclusion}

The interaction of $S$. aureus with osteoblasts is initiated from the attachment of the bacteria to osteoblasts, followed by the internalization of $S$. aureus by osteoblasts. Signal transduction and cytokine expression play a vital role in the process of inflammatory damage and destruction of the bone.

$S$. aureus, internalized in the osteoblasts, may be sequestered from the majority of antibiotics and the immune system. However, the sensitivity change of the bacteria to the antibiotics capable of penetrating eukaryotic cells results in the failure of antibiotic therapy. Therefore, metabolic characteristics of
S. aureus in the intracellular environment should be further studied to develop novel antibiotics to kill the bacteria in the osteoblasts. In addition, the internalization process should also be considered; if we are able to prohibit the invasion process and locate the bacteria outside the host cells, then eradication of these bacterias becomes simpler.

Osteoblasts challenged by $S$. aureus play a significant role in the initiation and maintenance of the immune response. The process is complicated and involves numerous cytokines and pathways that conduct the signal. Although recruitment of leukocytes and phagocytes may be helpful in killing the pathogens, inflammatory damage may accumulate. Apoptosis of osteoblasts induced by S. aureus and the increased activity of osteoclasts are responsible for bone resorption (39). Downregulation of cytokines may inhibit the destruction of bone; however, down-regulation of cytokines also inhibits the immune response, which may be helpful in the process of eradicating bacteria (32). Therefore, it appears more promising and easier to inhibit the attachment and internalization of $S$. aureus by osteoblasts in treating osteomyelitis. Further investigation into the interaction of $S$. aureus with osteoblasts should therefore be performed in order to decrease the rate of orthopedic infection.

\section{References}

1. Chihara S and Segreti J: Osteomyelitis. Dis Mon 56: 6-31, 2010.

2. Rao N, Ziran BH and Lipsky BA: Treating osteomyelitis: antibiotics and surgery. Plast Reconstr Surg 127 (Suppl 1): S177-S187, 2011.

3. Stocks $\mathrm{G}$ and Janssen HF: Infection in patients after implantation of an orthopedic device. ASAIO J 46: S41-S46, 2000.

4. Webb LX, Wagner W, Carroll D, Tyler H, Coldren F and Martin E: Osteomyelitis and intraosteoblastic Staphylococcus aureus. J Surg Orthop Adv 16: 73-78, 2007.

5. Brady RA, O'May GA, Leid JG, Prior ML, Costerton JW and Shirtliff ME: Resolution of Staphylococcus aureus biofilm infection using vaccination and antibiotic treatment. Infect Immun 79: 1797-1803, 2011.

6. Garzoni C and Kelley WL: Staphylococcus aureus: new evidence for intracellular persistence. Trends Microbiol 17: 59-65, 2009.

7. Ahmed S, Meghji S, Williams RJ, Henderson B, Brock JH and Nair SP: Staphylococcus aureus fibronectin binding proteins are essential for internalization by osteoblasts but do not account for differences in intracellular levels of bacteria. Infect Immun 69: 2872-2877, 2001

8. Alexander EH, Bento JL, Hughes FM, Marriott I, Hudson MC and Bost KL: Staphylococcus aureus and Salmonella enterica serovar dublin induce tumor necrosis factor-related apoptosisinducing ligand expression by normal mouse and human osteoblasts. Infect Immun 69: 1581-1586, 2001.

9. Gay CV, Gilman VR and Sugiyama T: Perspectives on osteoblast and osteoclast function. Poult Sci 79: 1005-1008, 2000.

10. Almeida RA, Matthews KR, Cifrian E, Guidry AJ and Oliver SP: Staphylococcus aureus invasion of bovine mammary epithelial cells. J Dairy Sci 79: 1021-1026, 1996.

11. Sinha B and Herrmann M: Mechanism and consequences of invasion of endothelial cells by Staphylococcus aureus. Thromb Haemost 94: 266-277, 2005.

12. Ellington JK, Reilly SS, Ramp WK, Smeltzer MS, Kellam JF and Hudson MC: Mechanisms of Staphylococcus aureus invasion of cultured osteoblasts. Microb Pathog 26: 317-323, 1999.

13. Jevon M, Guo C, Ma B, Mordan N, Nair SP, Harris M, Henderson B, Bentley G and Meghji S: Mechanisms of internalization of Staphylococcus aureus by cultured human osteoblasts. Infect Immun 67: 2677-2681 1999.

14. Wright JA and Nair SP: Interaction of staphylococci with bone. Int J Med Microbiol 300: 193-204, 2010.

15. Ellington JK, Harris M, Hudson MC, Vishin S, Webb LX and Sherertz R: Intracellular Staphylococcus aureus and antibiotic resistance: implications for treatment of staphylococcal osteomyelitis. J Orthop Res 24: 87-93, 2006. 
16. Reilly SS, Hudson MC, Kellam JF and Ramp WK: In vivo internalization of Staphylococcus aureus by embryonic chick osteoblasts. Bone 26: 63-70, 2000.

17. Bosse MJ, Gruber HE and Ramp WK: Internalization of bacteria by osteoblasts in a patient with recurrent, long-term osteomyelitis. A case report. J Bone Joint Surg Am 87: 1343-1347, 2005.

18. Fowler T, Wann ER, Joh D, Johansson S, Foster TJ and Höök M: Cellular invasion by Staphylococcus aureus involves a fibronectin bridge between the bacterial fibronectin-binding MSCRAMMs and host cell beta1 integrins. Eur J Cell Biol 79: 672-679, 2000.

19. Khalil H, Williams R, Stenbeck G, Henderson B, Meghji S and Nair S: Invasion of bone cells by Staphylococcus epidermidis. Microbes Infect 9: 460-465, 2007.

20. Nair SP, Bischoff M, Senn MM and Berger-Bachi B: The sigma B regulon influences internalization of Staphylococcus aureus by osteoblasts. Infect Immun 71: 4167-4170, 2003

21. Zhang Q, Feng Y, Zhou Q, Luo Q, Zhang X and Qin L: [Contribution of sigma $\mathrm{B}$ to environmental stress tolerance in Listeria monocytogenes - a review]. Wei Sheng Wu Xue Bao (In Chinese) 49: 1282-1288, 2009.

22. Hudson MC, Ramp WK, Nicholson NC, Williams AS and Nousiainen MT: Internalization of Staphylococcus aureus by cultured osteoblasts. Microb Pathog 19: 409-419, 1995.

23. Sinha B and Herrmann M: Mechanism and consequences of invasion of endothelial cells by Staphylococcus aureus. Thromb Haemost 94: 266-277 2005.

24. Finlay BB and Cossart P: Exploitation of mammalian host cell functions by bacterial pathogens. Science 276: 718-725, 1997.

25. Ellington JK, Elhofy A, Bost KL and Hudson MC: Involvement of mitogen-activated protein kinase pathways in Staphylococcus aureus invasion of normal osteoblasts. Infect Immun 69: 5235-5242, 2001

26. Pulverer BJ, Kyriakis JM, Avruch J, Nikolakaki E and Woodgett JR: Phosphorylation of c-jun mediated by MAP kinases. Nature 353: 670-674, 1991.

27. Rouzer CA, Matsumoto T and Samuelsson B: Single protein from human leukocytes possesses 5-lipoxygenase and leukotriene A4 synthase activities. Proc Natl Acad Sci USA 83: 857-861, 1986.

28. Pace J, Hayman MJ and Galan JE: Signal transduction and invasion of epithelial cells by S. typhimurium. Cell 72: 505-514, 1993.

29. Wilson CB, Jacobs RF and Smith AL: Cellular antibiotic pharmacology. Semin Perinatol 6: 205-213, 1982.

30. Ellington JK, Harris M, Hudson MC, Vishin S, Webb LX and Sherertz R: Intracellular Staphylococcus aureus and antibiotic resistance: implications for treatment of staphylococcal osteomyelitis. J Orthop Res 24: 87-93, 2006.

31. Ellington JK, Harris M, Webb L, et al: Intracellular Staphylococcus aureus. A mechanism for the indolence of osteomyelitis. J Bone Joint Surg Br 85: 918-921, 2003.

32. Marriott I: Osteoblast responses to bacterial pathogens: a previously unappreciated role for bone-forming cells in host defense and disease progression. Immunol Res 30: 291-308, 2004.

33. Bost KL, Ramp WK, Nicholson NC, Bento JL, Marriott I and Hudson MC: Staphylococcus aureus infection of mouse or human osteoblasts induces high levels of interleukin- 6 and interleukin-12 production. J Infect Dis 180: 1912-1920, 1999.

34. Trembleau S, Germann T, Gately MK and Adorini L: The role of IL-12 in the induction of organ-specific autoimmune diseases. Immunol Today 16: 383-386, 1995.
35. Marriott I, Gray DL, Rati DM, et al: Osteoblasts produce monocyte chemoattractant protein-1 in a murine model of Staphylococcus aureus osteomyelitis and infected human bone tissue. Bone 37: 504-512, 2005

36. Rossi D and Zlotnik A: The biology of chemokines and their receptors. Annu Rev Immunol 18: 217-242, 2000.

37. Gasper NA, Petty CC, Schrum LW, Marriott I and Bost KL: Bacterium-induced CXCL10 secretion by osteoblasts can be mediated in part through toll-like receptor 4. Infect Immun 70: 4075-4082, 2002.

38. Wright KM and Friedland JS: Regulation of chemokine gene expression and secretion in Staphylococcus aureus-infected osteoblasts. Microbes Infect 6: 844-852, 2004

39. McCall SH, Sahraei M, Young AB, et al: Osteoblasts express NLRP3, a nucleotide-binding domain and leucine-rich repeat region containing receptor implicated in bacterially induced cell death. J Bone Miner Res 23: 30-40, 2008.

40. Jin C and Flavell RA: Molecular mechanism of NLRP3 inflammasome activation. J Clin Immunol 30: 628-631, 2010.

41. Marriott I, Rati DM, McCall SH and Tranguch SL: Induction of Nod1 and Nod2 intracellular pattern recognition receptors in murine osteoblasts following bacterial challenge. Infect Immun 73: 2967-2973, 2005.

42. Le Bourhis L, Benko S and Girardin SE: Nod1 and Nod2 in innate immunity and human inflammatory disorders. Biochem Soc Trans 35: 1479-1484, 2007.

43. Giuliani C, Napolitano G, Bucci I, Montani V and Monaco F: [Nf- $\kappa \mathrm{B}$ transcription factor: role in the pathogenesis of inflammatory, autoimmune, and neoplastic diseases and therapy implications]. Clin Ter (In Italian) 152: 249-253, 2001

44. Ning R, Zhang X, Guo X and Li Q: Attachment of Staphylococcus aureus is required for activation of nuclear factor $\mathrm{\kappa B}$ in human osteoblasts. Acta Biochim Biophys Sin (Shanghai) 42: 883-892, 2010.

45. Tucker KA, Reilly SS, Leslie CS and Hudson MC: Intracellular Staphylococcus aureus induces apoptosis in mouse osteoblasts. FEMS Microbiol Lett 186: 151-156, 2000.

46. Alexander EH, Rivera FA, Marriott I, Anguita J, Bost KL and Hudson MC: Staphylococcus aureus - induced tumor necrosis factor - related apoptosis - inducing ligand expression mediates apoptosis and caspase- 8 activation in infected osteoblasts. BMC Microbiol 3: 5, 2003

47. Nair SP, Meghji S, Wilson M, Reddi K, White P and Henderson B: Bacterially induced bone destruction: mechanisms and misconceptions. Infect Immun 2371-2380, 1996.

48. Nair S, Song Y, Meghji S, et al: Surface-associated proteins from Staphylococcus aureus demonstrate potent bone resorbing activity. J Bone Miner Res 10: 726-734, 1995.

49. Hofbauer LC and Heufelder AE: Intercellular chatter: osteoblasts, osteoclasts and interleukin 6. Eur J Endocrinol 134 425-426, 1996

50. Ishida I, Kohda C, Yanagawa Y, Miyaoka H and Shimamura T: Epigallocatechin gallate suppresses expression of receptor activator of NF- $\mathrm{KB}$ ligand (RANKL) in Staphylococcus aureus infection in osteoblast-like NRG cells. J Med Microbiol 56 1042-1046, 2007.

51. Bost KL, Bento JL, Ellington JK, Marriott I and Hudson MC: Induction of colony-stimulating factor expression following Staphylococcus or Salmonella interaction with mouse or human osteoblasts. Infect Immun 68: 5075-5083, 2000. 